\title{
An unusual case of follicular dendritic cell sarcoma of the omentum with pleomorphous morphology and aberrant cytokeratin expression
}

\author{
Stefano Fratoni - Pasquale Niscola • Luca Cupelli • Andrea Tendas • \\ Laura Scaramucci • Luciana Morino • Teresa Dentamaro • \\ Paolo de Fabritiis • Giuseppe Santeusanio
}

Received: 2 August 2014 / Accepted: 26 August 2014 / Published online: 9 September 2014

(C) Springer-Verlag Berlin Heidelberg 2014

\begin{abstract}
Follicular dendritic cell sarcoma (FDCS) is an uncommon tumor arising from follicular dendritic cells (FDCs) in the lymph nodes or in extranodal sites. We herein report an unusual case of a 30-year-old man who presented with a FDCS of the omentum and massive liver involvement. The tumor proliferation was a diffuse growth composed by large and highly pleomorphic cells. On immunostaining, B and T cell markers; CD30, myeloid, and histiocytic markers; and S100 were negative whereas CD21, CD23, CD35, clusterin, and fascin were positive. Unexpectedly, large spectrum cytokeratin clone AE1/AE3 was focally and variably expressed. Despite the latter atypical finding and the misleading morphology, immunohistochemistry allowed for a conclusive diagnosis of FDCS.
\end{abstract}

Keywords Dendritic cells · Follicular dendritic cell sarcoma $\cdot$ Liver S Stem cell transplantation

\section{Introduction}

Follicular dendritic cell sarcoma (FDCS) is a rare neoplasm $[1,2]$ arising most commonly from follicular dendritic cells (FDCs) in the lymph nodes and extranodal sites, including intra-abdominal organs [3-5]. Among the latter, the omentum

\footnotetext{
S. Fratoni $\cdot$ G. Santeusanio

Department of Surgical Pathology, St. Eugenio Hospital, Piazzale

dell'Umanesimo, 10, 00144 Rome, Italy

P. Niscola $(\varangle) \cdot$ L. Cupelli $\cdot$ A. Tendas $\cdot$ L. Scaramucci $\cdot$

L. Morino $\cdot$ T. Dentamaro $\cdot$ P. de Fabritiis

Hematology Unit, St. Eugenio Hospital, Piazzale dell'Umanesimo,

10, 00144 Rome, Italy

e-mail:pniscola@gmail.com
}

as a primitive site, as recently observed by us, has been exceptionally reported $[6,7]$. FDCS often represents a diagnostic challenge [8-10]. Herein, we reported a rare case of extranodal FDCS, for which the omentum was supposed as the primary site. Our case presented an unusual morphology and phenotype which made the diagnostic process very challenging. The complete characterization of the neoplasm as FDCS was obtained by the sequential application of large immunohistochemical panels which showed the typical FDCS phenotype.

\section{Clinical history}

We herein report an unusual case of a 30-year-old man who presented with general malaise and a remarkable hepatomegaly. His laboratory profile was near normal, including viral hepatitis and HIV serologic tests and levels of humoral serological markers, such as alpha-fetoprotein, carcinoembryonic antigen, and CA19-9. An abdominal ultrasound showed a non-specific pathological mass extending from the fourth hepatic segment and inglobating all the upper abdominal structures, including the mesogastrium and the pancreas. In addition, a wide hepatic dissemination by pathological nodules was also present. The radiological aspects were consistent with a solid and compact mass, without internal areas of necrosis and/or calcifications; no cleavage planes were observable. These findings were confirmed by a body computerized tomography scan that ruled out other extra-abdominal localizations and providing findings highly suggestive for an omentum origin of the neoplastic process. The histopathological examination of bone marrow (BM) core biopsy showed no malignancy, so the patient underwent an excisional biopsy and a diagnosis of FDCS was made. Therefore, he was started 
on combination chemotherapy, receiving CHOP regimen, consisting of cyclophosphamide, doxorubicin, vincristine, and prednisone; the patient achieved only a partial remission. The treatment was then intensified, so that the patient received three cycles of dexamethasone, high-dose cytarabine, and cisplatin (DHAP) and then submitted to allogeneic stem cell transplantation from a family donor, achieving only a briefly transient tumor response. Therefore, in spite of salvage regimens including the gemcitabine-cisplatin combination and the tyrosine kinase inhibitor imatimib, the patient died because of progressive disease about 4 years from the primary diagnosis.

\section{Materials and methods}

Wright-Giemsa-stained peripheral blood and BM aspirate smears and trephine biopsy along with surgical specimens taken from the abdominal mass were received. Sections from abdominal tumor biopsy were fixed in $10 \%$ neutral buffered formalin. The BM biopsy was fixed in $10 \%$ neutral buffered formalin with subsequent decalcification. Specimens were routinely processed and embedded in paraffin using routine histological techniques. Section stains were performed on $2-\mu \mathrm{m}$ paraffin sections and subsequently stained with hematoxylin and eosin. BM aspirate preparations were stained with WrightGiemsa stain. Immunohistochemical stains were performed on $2-\mu \mathrm{m}$ paraffin sections of formalin-fixed, paraffin-embedded tissues; immunohistochemical stains used were CD79a, CD20, PAX5, CD3 CD2, CD5, CD138, kappa and lambda light chain, leucocyte common antigen (LCA), CD30, CD68KP1, CD68RPGM1, CD163, S100, CD1a, large spectrum cytokeratin AE1/ AE3, myeloperoxidase (MPO), CD34, CD117, CD43, CD56, CD21, CD23, CD35, clusterin, and fascin. The proliferative index was evaluated with Ki67MIB1.

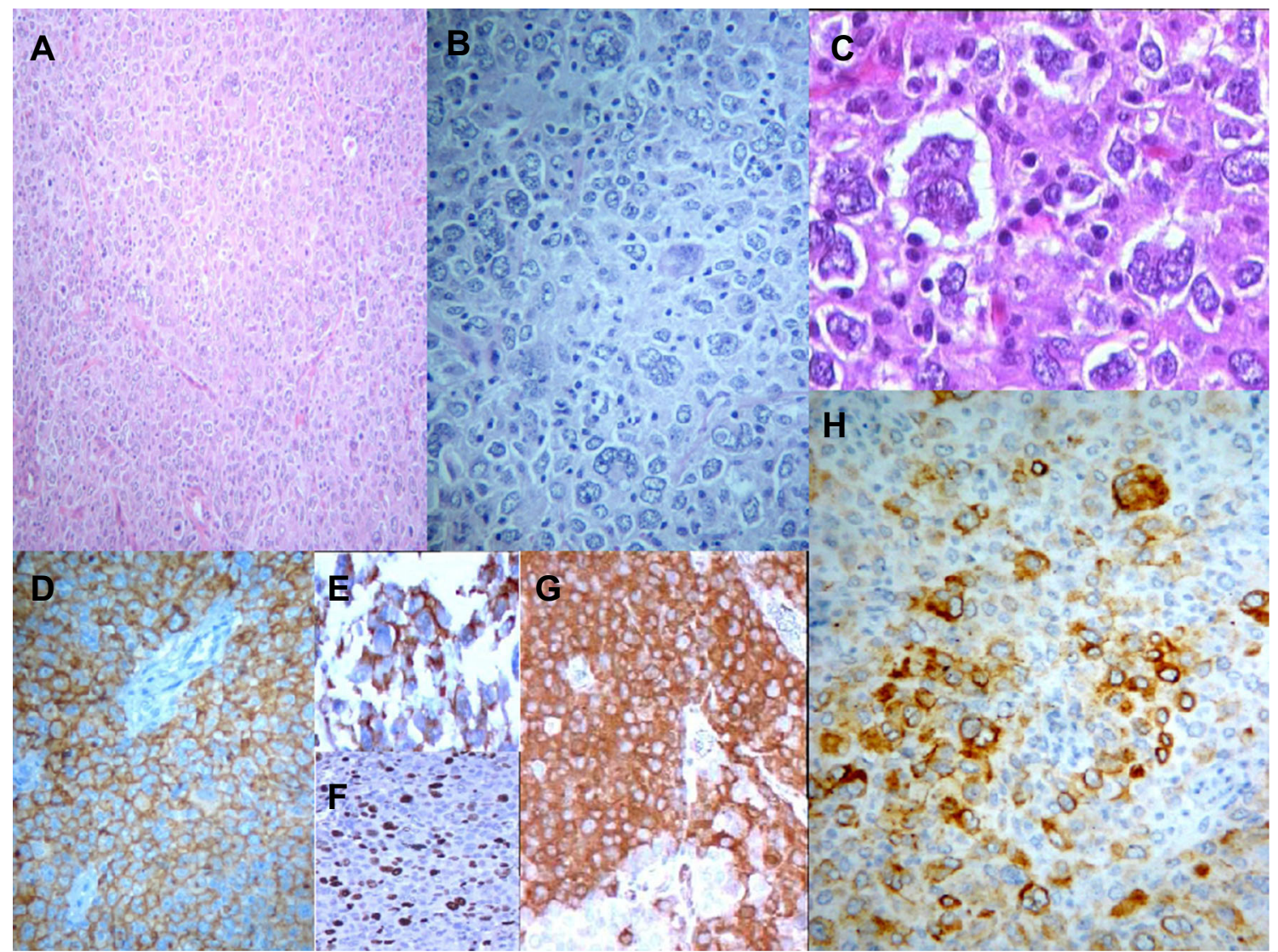

Fig. 1 a Magnification $(\times 10)$ shows a neoplastic population with a diffuse pattern of growth, composed predominantly by large pleomorphic ovoid cells with eosinophilic cytoplasm with syncytial features. b Medium magnification $(\times 20)$ clearly reveals the pleomorphism of the neoplastic population which gets large polilobulated nuclei with vesicular chromatin but inconspicuous nucleoli. c High magnification $(\times 40)$ shows large cells with bizarre high-grade nuclear pleomorphism and eosinophilic cytoplasm. Some cells show some so-called kissing-like feature represented by two cells with closely packed nuclei. d, e, $\mathbf{g}$ Immunostains for follicular dendritic cell markers CD21 (d), CD35 (e), and fascin (g) were expressed. h Immunostain for large spectrum cytokeratin clone AE1/AE3 revealed focal and variable expression in several clusters of neoplastic cells. f Immunostain for Ki67MIB1 shows a proliferative fraction about $30 \%$ 


\section{Results}

The PB smears showed no abnormal findings; the BM was $90 \%$ cellular without any features compatible with dyspoiesis and/or tumor involvement. The pathological examination of the tumor specimen showed (Fig. 1a, $b, c)$ a neoplastic population with a diffuse pattern of growth, predominantly composed by undifferentiated, large, highly pleomorphic, and bizarre plump ovoid cells; the latter cells showed an eosinophilic cytoplasm with syncytial quality and atypical nuclei with vesicular chromatin and small distinct nucleoli. Scattered multinucleate tumor cells were also present. Many mitoses including atypical mitotic figures were readily demonstrated. Rare foci of coagulative necrosis were also present. On immunohistochemical analysis, CD79a, CD20, PAX5, CD3 CD2, CD5, CD138, kappa and lambda light chain, LCA, CD30, CD68KP1, CD68RPGM1, CD163, S100, and CD1a were negative. Unexpectedly, large spectrum cytokeratin clone AE1/ AE3 was focally expressed on several clusters of neoplastic cells. At first impression, this last immunohistochemical finding was interpreted as compatible with a high-grade neoplasm of epithelial derivation such as an undifferentiated carcinoma. However, the presence of abundant eosinophilic cytoplasm raised the hypothesis of the possible involvement of a myeloid or accessory cell/histiocytic/dendritic cell tumor. The proliferative index evaluated with Ki67MIB1 was about $30 \%$ (Fig. 1f). Therefore, an additional immunohistochemical panel including myeloid and FDCs related markers was performed. Then, the results revealed that myeloperoxidase, CD34, CD117, CD43, and CD56 were negative whereas CD21, CD23, CD35, fascin (Fig. 1d, e, g), and clusterin were diffusely expressed by tumor cells. On the basis of these immunohistochemical findings, despite the unusual and misleading morphology of the neoplastic cells, a diagnosis of FDCS was made.

\section{Discussion}

We describe here an unusual case of FDCS which gets some uncommon and interesting clinical-pathological features. FDCS is a rare, low-intermediate malignant potential tumor $[1,2]$ arising from non-lymphoid, nonphagocytic accessory follicular dendritic cells of the lymphoid follicles. FDCS mostly involves lymph nodes and extranodal sites, such as the oral cavity, tonsils, pharynx, and mediastinum as well as intra-abdominal organs $[1,2]$, representing the omentum, as our peculiar case does, an exceptionally rare primary site [6, 7]. Moreover, by pathological point of view, the present case shows aggressive histological features such as very atypical large plump ovoid cells along with bizarre pleomorphic nuclei, frequent atypical mitoses, and rare foci of tumor necrosis, which represent other atypical findings quite rarely observed in FDCS. As a matter of fact, classic finding of the tumor as well as spindle cell features and fascicular pattern of growth were totally absent. Finally, the aberrant expression of large spectrum cytokeratin AE1/AE3 by several clusters of FDCS cells is quite exceptional. Together, these pathological findings represented very challenging features which at first impression might suggest an incorrect diagnosis of high-grade undifferentiated pleomorphic carcinoma. Nevertheless, the diffuse expression of follicular dendritic cell markers was typical for FDCS. Indeed, FDCS may mimic a large spectrum of tumors such as undifferentiated carcinoma, pleomorphic sarcoma, thymoma, and gastrointestinal stromal tumor, so that the differential diagnosis from other malignancy may be so extensive that a very large immunohistochemical panel, including markers for accessory histiocyte/follicular dendritic cell markers, is necessary in order to define the correct cellular lineage [1, 4, 8-10]. As a matter of fact, every surgical pathologist/hematopathologist should be aware of including the FDCS into the differential diagnosis of any tumor with undifferentiated morphology composed by large "epithelioid polygonal" pleomorphic cells and large eosinophilic cytoplasm.

FDCS has been increasingly reported in recent years, although mostly in the form of single case reports or small case series so that its natural history and prognostic factors are not well understood as well as the most appropriate treatment is not yet well defined [1].

Our patient had a fatal disease. We supposed that the anatomical site and highly aggressive histopathological features may have been poor diagnostic factors. Indeed, the intraabdominal omentum as a primary site may be related to tumor asymptomatic overgrowth for a long time as well as to delayed diagnosis. In addition, the prominent cellular pleomorphism, the high mitotic rate with atypical figures, and foci of necrosis represent all features potentially related to an aggressive clinical behavior. In conclusion, we have reported a rare FDCS of the omentum with uncommon clinicopathological findings, aggressive histological features, and exceptional aberrant phenotype such as cytokeratin expression. Familiarity with these insidious and misleading findings is very important to differentiate this tumor from other malignancies to do a correct diagnosis for relevant therapy.

Conflict of interest The authors have no competing interests to disclose. No writing assistance was utilized in the production of this manuscript. 


\section{References}

1. Pileri SA, Grogan TM, Harris NL, Banks P, Campo E, Chan JK, Favera RD, Delsol G, De Wolf-Peeters C, Falini B, Gascoyne RD, Gaulard P, Gatter KC, Isaacson PG, Jaffe ES, Kluin P, Knowles DM, Mason DY, Mori S, Müller-Hermelink HK, Piris MA, Ralfkiaer E, Stein H, Su IJ, Warnke RA, Weiss LM (2002) Tumours of histiocytes and accessory dendritic cells: an immunohistochemical approach to classification from the International Lymphoma Study Group based on 61 cases. Histopathology 41(1):1-29

2. Denning KL, Olson PR, Maley RH Jr, Flati VR, Myers JL, Primary SJF, Kairouz S, Hashash J, Kabbara W, McHayleh W, Tabbara IA (2007) Dendritic cell neoplasms: an overview. Am J Hematol 82: 924-928

3. Shek TW, Liu CL, Peh WC, Fan ST, Ng IO (1998) Intra-abdominal follicular dendritic cell tumour: a rare tumour in need of recognition. Histopathology 33(5):465-470

4. Kang TW, Lee SJ, Song HJ (2010) Follicular dendritic cell sarcoma of the abdomen: the imaging findings. Korean J Radiol 11:239-243
5. Padilla-Rodríguez AL, Bembassat M, Lazaro M, Ortiz-Hidalgo C (2007) Intra-abdominal follicular dendritic cell sarcoma with marked pleomorphic features and aberrant expression of neuroendocrine markers: report of a case with immunohistochemical analysis. Appl Immunohistochem Mol Morphol 15(3):346-352

6. Chien JC, Lao WT, Chen CL, Chan WP (2013) Follicular dendritic cell sarcoma of the omentum: multidetector computed tomography findings. Korean J Radiol 14(2):213-217

7. Yamakawa M, Andoh A, Masuda A, Miyauchi S, Kasajima T, Ohmori A, Oguma T, Takasaki K (2002) Follicular dendritic cell sarcoma of the omentum. Virchows Arch 440(6):660-663

8. Malik A, Veniyoor A, Fanthome B, Dutta V (2012) Follicular dendritic cell sarcoma: a diagnostic challenge ! J Cancer Res Ther 8(2): 306-307

9. Orii T, Takeda H, Kawata S, Maeda K, Yamakawa M (2010) Differential immunophenotypic analysis of dendritic cell tumours. J Clin Pathol 63:497-503

10. Romero-Guadarrama MB, Reyes-Posada $\mathrm{O}$, Hernández-González MM, Durán-Padilla MA (2009) Follicular dendritic cell sarcoma/ tumor: 2 cases of a rare tumor of difficult clinical and pathological diagnosis. Ann Diagn Pathol 13(4):257-262 